Draft version March 13, 2022

Preprint typeset using $\mathrm{LAT}_{\mathrm{E}} \mathrm{X}$ style emulateapj v. 08/22/09

\title{
SOLAR MAGNETIC FIELD SIGNATURES IN HELIOSEISMIC SPLITTING COEFFICIENTS
}

\author{
Charles S. Baldner \\ Department of Astronomy, Yale University, P.O. Box 208101, New Haven, CT, 06520-8101 \\ H. M. Antia \\ Tata Institute of Fundamental Research, Homi Bhabha Road, Mumbai 400005, India \\ SARBANI BASU \\ Department of Astronomy, Yale University, P.O. Box 208101, New Haven, CT, 06520-8101 \\ AND \\ Timothy P. LARSON \\ Hansen Experimental Physics Laboratory, Stanford University, Stanford, CA 94305-4085 \\ Draft version March 13, 2022
}

\begin{abstract}
Normal modes of oscillation of the Sun are useful probes of the solar interior. In this work, we use the even-order splitting coefficients to study the evolution of magnetic fields in the convection zone over solar cycle 23, assuming that the frequency splitting is only due to rotation and a large scale magnetic field. We find that the data are best fit by a combination of a poloidal field and a doublepeaked near-surface toroidal field. The toroidal fields are centered at $r_{0}=0.999 R_{\odot}$ and $r=0.996 R_{\odot}$ and are confined to the near-surface layers. The poloidal field is a dipole field. The peak strength of the poloidal field is $124 \pm 17 \mathrm{G}$. The toroidal field peaks at $380 \pm 30 \mathrm{G}$ and $1.4 \pm 0.2 \mathrm{kG}$ for the shallower and deeper fields respectively. The field strengths are highly correlated with surface activity. The toroidal field strength shows a hysteresis-like effect when compared to the global $10.7 \mathrm{~cm}$ radio flux. The poloidal field strength shows evidence of saturation at high activity.

Subject headings: Sun: activity, Sun: helioseismology, Sun: magnetic fields
\end{abstract}

\section{INTRODUCTION}

Understanding the nature of the Sun's magnetic fields - their structure and variability, their generation mechanisms, and their effects on the heliosphere - is one of the key aims of current research in solar physics. It is generally believed that the magnetic fields are generated by a cyclic dynamo that operates somewhere in the solar interior. In this paper, we use helioseismology to study the global scale internal magnetic fields over the course of solar cycle 23 .

Helioseismology is the most powerful tool available to solar physicists to study the interior of the Sun. The oscillation frequencies have been used to study the structure and dynamics of the solar interior with great precision. Magnetic fields, however, have proved to be much more challenging. There are a number of important difficulties in dealing with magnetic fields in a helioseismic context. The magnitudes of the signatures in the data are quite small, making statistically significant measurements challenging. Secondly, the interpretation of data is very difficult. The physics of wave propagation in the presence of magnetic fields is far more complex than in the non-magnetic case. Further, the geometry of the underlying field strongly affects the signatures in helioseismic global mode frequencies, meaning different field configurations and strengths can be difficult to distinguish from their helioseismic signatures. Even worse, Zweibel \& Gough (1995) showed that because magnetic

Electronic address: charles.baldner@yale.edu fields act on mode frequencies both by perturbing the thermal structure of the Sun and by changing the wave propagation speeds directly, there is a degeneracy between magnetic field effects and other thermal perturbations which cannot be distinguished a priori from helioseismic data.

Although helioseismic determinations of magnetic fields are difficult, there have been many attempts to do so. Isaak (1982) suggested that the then observed frequency splittings in the solar acoustic spectrum could be caused by a large scale magnetic field situated in the core. Dziembowski \& Goode (1984) used an asymptotic approximation to study the effects of magnetic field on the splitting coefficients, and Dziembowski \& Goode (1988) argued that a 1 MegaGauss field at the base of the convection zone was necessary to explain the observed splitting coefficients. However, Basu (1997); Antia et al. (2000) placed a limit of 0.3 MG on the field at the base of the convection zone; thus the situation was unclear. A mega-Gauss magnetic field is also inconsistent with dynamo theories and constraints from other observations (e.g., D'Silva \& Choudhuri 1993).

Gough \& Thompson (1990) developed a formalism to compute the effects of rotation and axisymmetric magnetic fields on the frequency splittings (discussed in the following section), which Antia et al. (2000) used to analyze the first year of Michelson Doppler Imager (MDI) data. They placed limits on the strengths of internal toroidal fields, finding a limit of $20 \mathrm{kG}$ at a depth of $30 \mathrm{Mm}$, and a limit of $300 \mathrm{kG}$ at the base of the con- 
vection zone $\left(r=0.713 R_{\odot}\right)$. Dziembowski et al. (2000) inverted the mean frequencies and splitting coefficients for changes in temperature, and found that the resulting temperature perturbation could be explained by a change in magnetic field of $60 \mathrm{kG}$ at a depth of $45 \mathrm{Mm}$ $\left(r \sim 0.93 R_{\odot}\right)$.

Dziembowski et al. (2001) found that changes in $f$ mode frequencies from solar minimum to solar maximum implied a decrease in solar radius with activity, which they associated with a change between 4 and $8 \mathrm{Mm}$ in depth. In explaining this result with changing magnetic fields, they assumed a tangled field, but even so the magnitude of the change in field strength was strongly dependent on the radial distribution of the field. The change they required was $7 \mathrm{kG}$ for a uniform field, or substantially less $(1 \mathrm{kG}$ at $8 \mathrm{Mm})$ for an inwardly increasing field. Chou \& Serebryanskiv (2002, 2005) looked for signatures of a change at the base of the convection zone from low activity to high activity, and found signs of a small change, which they proposed could be due to a change in magnetic field of $170-290 \mathrm{kG}$. Baldner \& Basu (2008), working with an entire solar cycle's worth of helioseismic data, found a change in sound speed between solar maximum and solar minimum at the base of the convection zone, which, if due to a change in magnetic field, could indicate a change in field strength of $290 \mathrm{kG}$ at that depth.

In this work, we exploit the fact that we have much more helioseismic data than previous investigators had access to, and try to get a coherent picture of sub-surface solar magnetic fields and their temporal evolution. We extend the work of Antia et al. (2000), who considered toroidal magnetic fields, to include poloidal fields. This means that we can, in principle, consider any axisymmetric magnetic field configuration. We compute the effects of a wide variety of magnetic field configurations on the $a_{2}$ splitting coefficients, and compare them to a solar cycle's worth of MDI data. It is not clear if the solar magnetic field has large scale structure of the form we assume or whether it is in tangled state due to turbulence in the convection zone. Since the effect of magnetic field manifests through a quadratic term in magnetic field, our estimate may also be applicable to tangled field with some degree of approximation.

\section{PERTURBATIONS TO SOLAR OSCILLATION FREQUENCIES}

The frequencies of normal modes of oscillation $\nu_{n \ell m}$ are degenerate in $m$ in the case of a spherically symmetric star. Departures from spherical symmetry lift this degeneracy. When the departures from spherical symmetry are small, as they are in the case of the Sun, the differences in frequency for different values of $m$ will be small, and it is natural therefore to express the normal mode frequencies in terms of the mean frequency of the multiplet $\nu_{n \ell}$ and splitting coefficients $a_{j}$ :

$$
\nu_{n \ell m}=\nu_{n \ell}+\sum_{j=1}^{j_{\max }} a_{j}(n, \ell) \mathcal{P}_{j}^{(\ell)}(m)
$$

As is common in the current literature, the polynomials $\mathcal{P}_{j}^{(\ell)}(m)$ are the Ritzwoller-Lavely formulation of the Clebsch-Gordan expansion (Ritzwoller \& Lavely 1991).
The odd-order splitting coefficients are caused by the rotation of the Sun, and will not be directly considered in this work. The even-order coefficients are caused by second order effects of rotation, and by the effects of magnetic fields or any other departure from spherical symmetry in the solar structure. In this work, we treat rotation and magnetic fields as perturbations on the spherically symmetric case, which allows us to avoid explicitly constructing a model of a rotating, magnetized star. The formalism was developed by Gough \& Thompson (1990) and Antia et al. (2000) extended the formalism to include the perturbation to the gravitational potential (i.e., to relax the Cowling approximation) and to include differential rotation.

The first order correction to the mode frequencies due to rotation affects only the odd-order splitting coefficients. These effects are due to the perturbation of the mode frequencies by advection of the waves. The second order correction affects only the even-order splitting coefficients, and is caused by the perturbation to the eigenfunctions and the centrifugal force. The odd-order coefficients can be used to determine the rotation profile $\Omega(r)$ (Thompson et al. 1996; Schou et al. 1998), which can in turn be used to compute the second order rotation correction (Antia et al. 2000) to the even-order coefficients. This correction needs to be made if the magnetic perturbation is comparable in size to second order rotation effect, which appears to be the case (Gough \& Thompson 1990; Antia et al. 2000).

In this work, we consider two different axisymmetric magnetic field configurations: toroidal and poloidal. Following Gough \& Thompson (1990), the toroidal field is expressed in the form

$$
\boldsymbol{B}=\left[0,0, a(r) \frac{d}{d \theta} P_{k}(\cos \theta)\right]
$$

where $P_{k}$ are the Legendre polynomials of degree $k$ and $a(r)$ describes the radial profile of the magnetic field. We consider only even values of $k$ to ensure antisymmetry about the equator, consistent with the observed field at the surface. The poloidal field is assumed to be of the form

$$
\boldsymbol{B}=\left[k(k+1) \frac{b(r)}{r^{2}} P_{k}(\cos \theta), \frac{1}{r} \frac{d b}{d r} \frac{d}{d \theta} P_{k}(\cos \theta), 0\right],
$$

where $b(r)$ describes the radial profile of the magnetic field. In this case we use only odd values of $k$ to ensure that the field is antisymmetric about the equator. With appropriate combinations of these two fields we can, in principal, represent any axisymmetric magnetic field.

The effect of these magnetic field configurations on the frequency splittings of $p$-modes is calculated using the formulation of Gough \& Thompson (1990); Antia et al. $(2000)$. There are two ways in which the magnetic field can affect the frequencies, one is the so-called direct effect due to the additional force, and the second is the distortion effect due to the equilibrium state being distorted from the original spherically symmetric one. Both these effects are included in all calculations. These formulations treat the effect of these magnetic fields separately. Unfortunately, the effect of magnetic fields is not linear and hence strictly the contributions from two different configurations cannot be added. In principle, there will 
be some cross-terms when the combination of toroidal and poloidal fields have a region of overlap in the solar interior. In this work, we neglect these terms and add the contributions from toroidal and poloidal fields to get the total effect. We expect the cross terms to be small.

\section{DATA}

The data we use for comparison are 72-day mode parameter sets from the Michelson Doppler Imager (MDI) on the SOlar and Heliospheric Observatory (SOHO). We use mode parameter sets from the corrected pipeline described by Larson \& Schou (2008). The original MDI analysis pipeline (Schou 1999) did not take in to account a number of instrumental effects which introduced secular trends in the mode parameter sets. In particular, the plate scale of the MDI instrument has changed somewhat over SOHO's mission, and this results in an apparent change in the solar radius if not properly corrected in the analysis. Baldner \& Basu (2008) found a signature in the mean frequencies which became increasingly significant over the course of the solar cycle. A repetition of that work with reanalyzed mode parameter sets removed this effect completely (Baldner et al. 2008). The splitting coefficients, which we focus on in this work, suffer from similar instrumental effects as the mean frequencies, and hence we use the reanalyzed data in this work.

We include 56 mode sets which cover solar cycle 23 . The mode sets are identified by the MDI start day, beginning with set \#1216 (start day 1 May 1996), and ending with set \#5320 (start day 27 July 2007). The coverage begins and ends at low activity, with a $10.7 \mathrm{~cm}$ radio flux of 72.7 SFU for the first set and a flux of 69.1 SFU for the last set. The highest activity set, \#3160 (start day 27 August 2001), has a $10.7 \mathrm{~cm}$ flux of 223.9 SFU.

We fit only the $a_{2}$ splitting coefficients, as the higher order splitting coefficients have larger errors, and as such did not distinguish well between different field configurations. The rotation profile determined from the oddorder splitting coefficients (Antia et al. 2008) was used to calculate the second-order contribution to the evenorder coefficients, and this contribution was subtracted from the data.

\section{RESULTS}

\subsection{Models}

In Fig. 11 we show the second splitting coefficient for four different poloidal field configurations. The actual quantities plotted are $\ell a_{2}$, both as a function of frequency $\nu$ and as a function of the lower turning radius of the modes, $r_{t}$. The radial profile in this case is taken to be

$$
b(r)=B_{0} r^{-k}
$$

where $B_{0}$ is a constant which determines the peak field strength and $r$ is the radial distance measured in units of solar radius. The models shown in Fig. 1 all have a peak strength of $B=1 \mathrm{G}$ (note that they do not all have the same value of $B_{0}$ ). The most obvious difference between different order poloidal fields is that for the $k=1$ field the splitting coefficients are all positive, whereas for the higher order fields they are largely negative, although the shallow modes have positive $a_{2}$.

The toroidal field we employ is similar to that used by

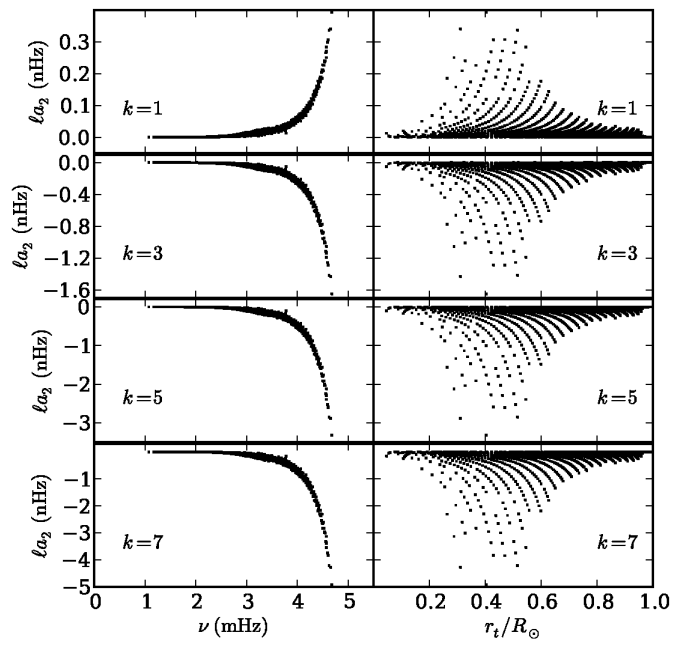

FIG. 1.- Splitting coefficients $\ell a_{2}$ due to poloidal magnetic fields. The left hand panels are shown as a function of frequency $\nu$, the right hand panels are shown as a function of lower turning radius $r_{t}$. The four configurations shown have peak field strengths of $1 \mathrm{G}$ at the surface. The fields have four different values of $k$. To facilitate direct comparison with later figures, only modes measured in the MDI data (specifically, the high activity set \#3160) are plotted.

Antia et al. (2000), with a radial profile given by

$$
a(r)= \begin{cases}\sqrt{8 \pi p \beta_{0}}\left(1-\left(\frac{r-r_{0}}{d_{0}}\right)^{2}\right) & \text { if }\left|r-r_{0}\right| \leq d_{0} \\ 0 & \text { otherwise }\end{cases}
$$

where $p$ is the gas pressure, $\beta_{0}$ is the ratio of the magnetic to gas pressure at $r_{0}$, and $r_{0}$ and $d_{0}$ are position and width of the field. As is the case for the poloidal fields, the toroidal field corrections are linear in magnetic field strength squared. Excepting field strength, therefore, our toroidal fields are described by three quantities: the order of the Legendre polynomial $k$, which determines the latitudinal distribution of the field, the central radius $r_{0}$, which determines the location, and the width $d_{0}$. Figure 2 shows the splitting coefficients due to toroidal fields with different values of $k$ but the same radial profile (in this case, $\beta_{0}=10^{-4}, r_{0}=0.999 R_{\odot}$ and $\left.d_{0}=0.001 R_{\odot}\right)$. For the $a_{2}$ coefficient, the order $k$ of the field makes very little difference except to the scale of the perturbation - increasing $k$ for the same $\beta_{0}$ effectively increases the total amount of flux, but except for this effect, the $a_{2}$ coefficients are not sensitive to different latitudinal distributions. For the remainder of the work, therefore, we restrict ourselves to $k=2$ fields.

Figure 3 shows the splitting coefficients $\ell a_{2}$ for nearsurface toroidal fields with different central radii $r_{0}$ and widths $d_{0}$ as a function of frequency. Figure 4 shows the same, but as a function of the lower turning radius, $r_{t}$. The behavior of the splitting coefficients is not surprising. In general, the fields which penetrate below the surface show oscillatory behavior as a function of frequency similar to that seen in mode frequency (Gough 1990; Gough \& Thompson 1990) and used by Roxburgh \& Vorontsov (1994); Basu et al. (1994) and others to study the convection zone base. The period of these oscillations is related to the acoustic depth of the perturbation in the structure. Decreasing the depth of 

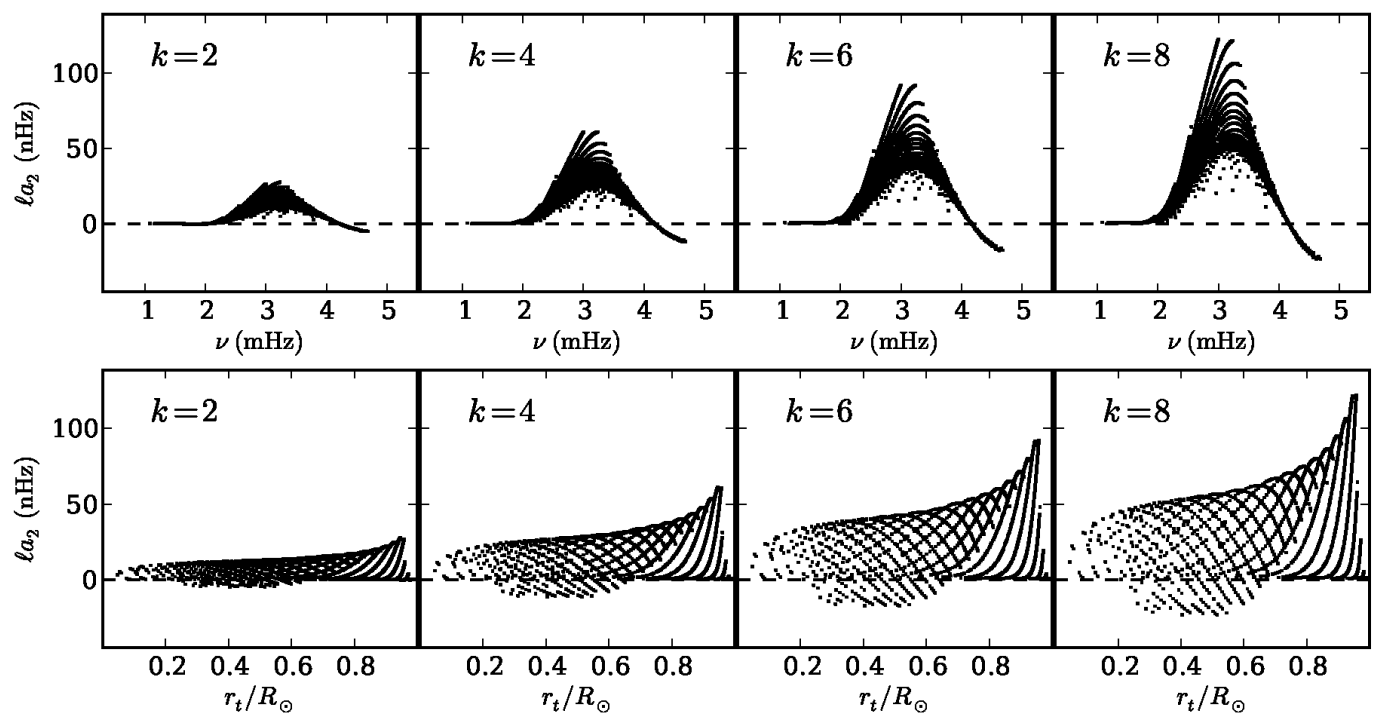

FIG. 2.- Splitting coefficients $\ell a_{2}$ due to toroidal field with different latitudinal distributions. The upper panels show the coefficients as a function of frequency $\nu$, the lower panels show the coefficients as a function of lower turning radius $r_{t}$. All the results are with $\beta_{0}=10^{-4}$, $r_{0}=0.999 R_{\odot}$ and $d_{0}=0.001 R_{\odot}$. Only modes present in the MDI data have been plotted.

the perturbation lengthens the period of the oscillatory behavior. Fields which are confined near the surface, on the other hand, do not exhibit oscillatory behavior, but instead resemble the 'surface term' correction which is removed in structure inversions (e.g., Dziembowski et al. 1990; Antia \& Basu 1994). Increasing the width of the perturbation smears out the oscillatory signature, as seen in Fig. 3. Because all the modes sampled have lower turning radii below the magnetic fields considered here, there are no obvious signatures in the splitting coefficients as a function of $r_{t}$.

In addition to fields near the surface, in Fig. 5, we show the splitting coefficients due to some toroidal fields located at the base of the convection zone. The fields shown differ only in the width $d_{0}$ of the fields. Unlike the surface fields shown in previous figures, the deep field signatures show both positive and negative splitting coefficients. These models are most interesting as a function of lower turning radius $r_{t}$. The splitting coefficients are positive above the center of the magnetic field, and negative below the center of the magnetic field. Further, as the width of the field is increased, the width of the perturbations to the splitting coefficients (in $r_{t}$ figure) increases as well.

The $a_{4}$ splitting coefficients due to various poloidal and toroidal fields are shown in Fig. 6, which shows the results for two poloidal fields, the $k=3$ and $k=7$ fields, as well as two toroidal fields with different values of $k(k=2$ and $k=8$ ), each with $r_{0}=0.999 R_{\odot}$ and $d_{0}=0.001 R_{\odot}$. The $k=1$ field has essentially no effect on the $a_{4}$ splitting coefficients.

\subsection{Fits to observed data}

In order to choose the fields which best match the actual data, we have computed the splitting coefficients for a large grid of field configurations, with fields throughout the convection zone. For poloidal fields we varied $k$ - the form of the radial profile was found not to matter very much for the splitting coefficients, so long as the field penetrated below the surface. For the toroidal fields, we varied the location $r_{0}$, the width of the field $d_{0}$, and the latitudinal distribution with $k$. The range in $r_{0}$ was between $0.70 R_{\odot}$ and $1.0 R_{\odot}$. The values for $d_{0}$ ranged from $10^{-4} R_{\odot}$ to $0.2 R_{\odot}$. In order to judge goodness-offit, we use the $\chi^{2}$ statistic. For both the poloidal and the toroidal fields, the perturbations vary linearly with the square of the field strength, so to fit the field, we allowed the field strength to vary freely, and chose the strength that minimized the $\chi^{2}$. We have computed the $\chi^{2}$ for all the field configurations in our grid, as well as for many combinations of two and three different fields. The results we present below represent the best fits from the entire grid of computed models.

The largest signal-to-noise ratio in $a_{2}$ is found at peak activity, and so the highest activity set ought to be the easiest to fit. Comparison of different field configurations with the splitting coefficients at high activity are shown in Fig. 7] and the fits are shown both as a function of frequency and as a function of lower turning radius. The residuals, normalized by the errors in the data, are also shown. A fit to a $k=1$ poloidal field is shown in panel (a). The reduced $\chi^{2}$ for this fit is 16 , and it is evident that the field does a poor job of reproducing the observed splitting coefficients. Higher order poloidal fields are considerably worse, as an examination of Fig. 1 will show - these fields perturb all the splitting coefficients negatively, whereas the observed splittings are all positive. Panel (b) shows the effect of a toroidal field situated near the surface. Although we attempted to fit toroidal fields throughout the convection zone, fields not located very near the surface were extremely poor fits to the data. The field shown in panel (b) is the best fit for a single toroidal field, with $r_{0}=0.999 R_{\odot}$ and $d_{0}=0.001 R_{\odot}$. The reduced $\chi^{2}$ is 5 . The residuals are mostly without structure in $r_{t}$, but are oscillatory in frequency, a hint that there could be a second, somewhat deeper field. The splitting coefficients at peak solar activity cannot be well fit by either a toroidal field or a poloidal field of the form considered by us.

The third field configuration shown (panel c) is a com- 


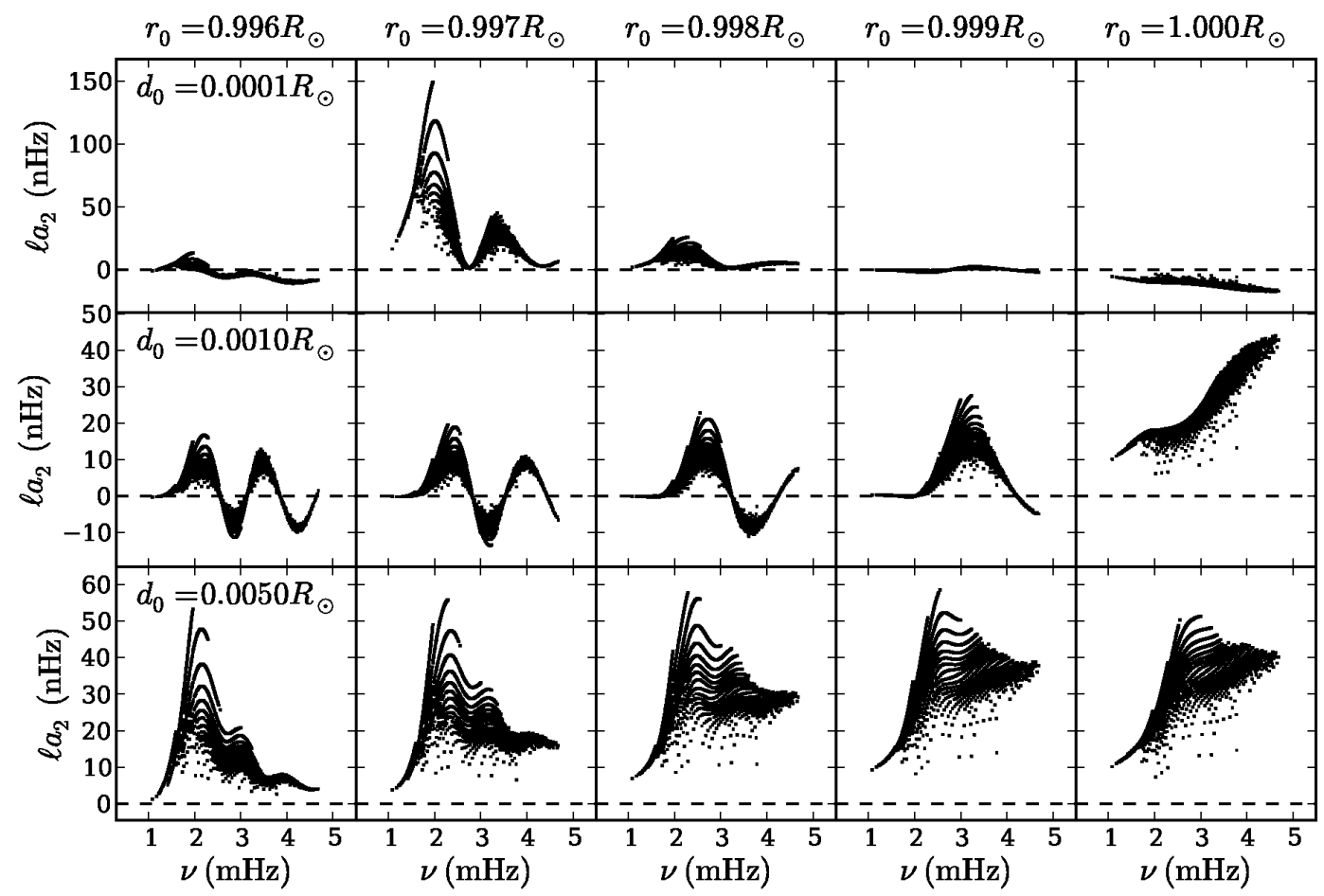

FIG. 3.- Splitting coefficients $\ell a_{2}$ due to near-surface toroidal magnetic fields, as a function of frequency $\nu$. The results are shown for $k=2$ with five different values of central radius $r_{0}$ (from $0.996 R_{\odot}$ to $R_{\odot}$ ), and three different values of the width of the field $d_{0}$ $(0.0001,0.001$, and 0.005$) R_{\odot}$. Only modes present in the MDI data have been plotted.

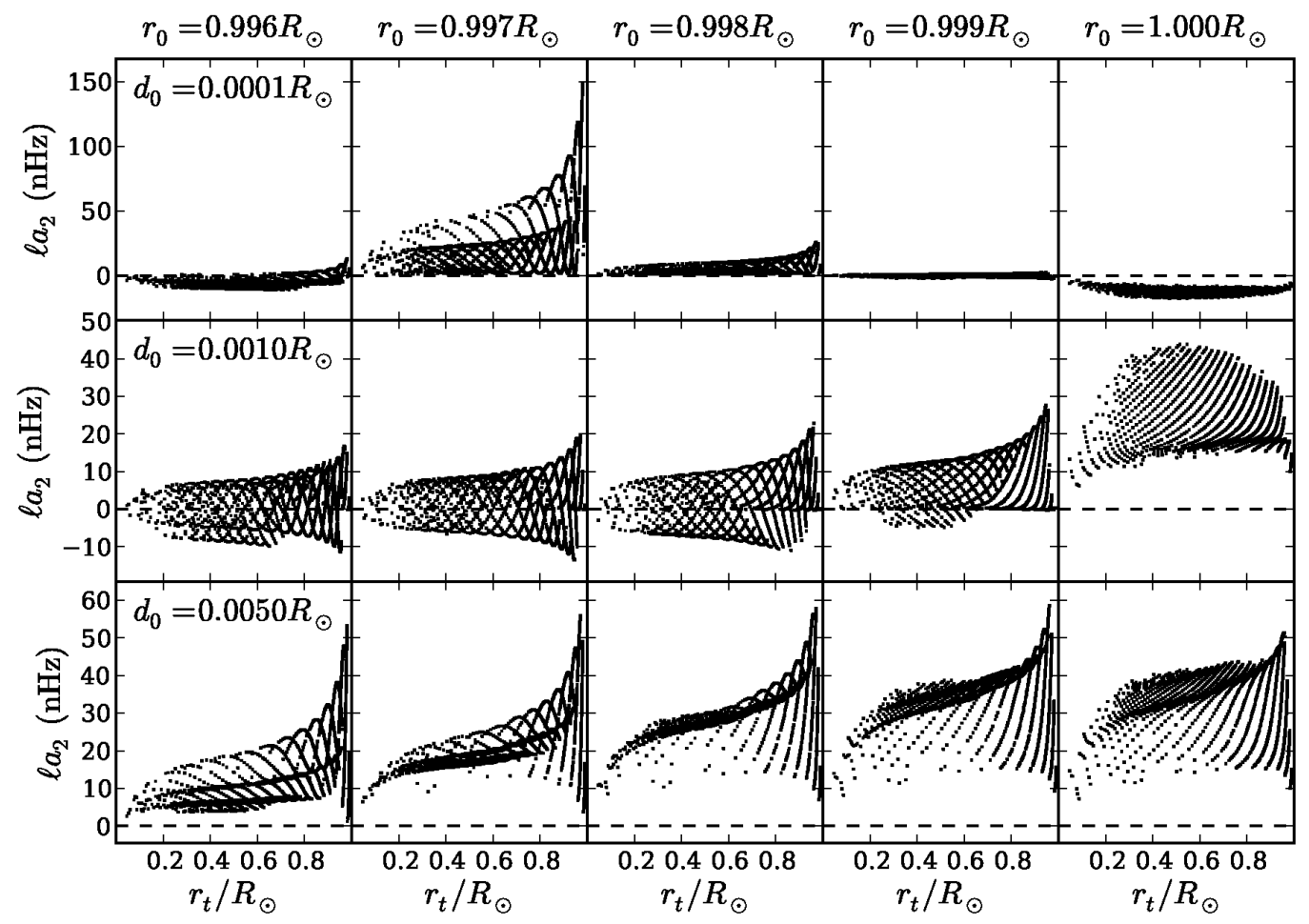

FIG. 4.- Same as Fig. 3 but plotted as a function of the lower turning radius $r_{t}$. 


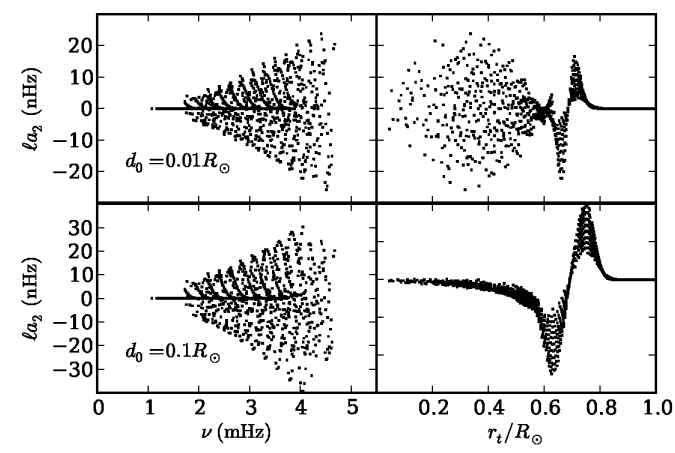

FIG. 5.- Effects of toroidal magnetic fields at the base of the convection zone on the $\ell a_{2}$ splitting coefficients. Results for two magnetic field configuration with $k=2, r_{0}=0.71 R_{\odot}$ and $\beta_{0}=10^{-4}$ are shown. The fields have widths of $d_{0}=0.01 R_{\odot}$ and $d_{0}=0.1 R_{\odot}$ for the top and bottom panels, respectively. Left hand panels show the splitting coefficients as a function of frequency $\nu$, right hand panels show the splitting coefficients as a function of lower turning radius $r_{t}$. Only observed modes have been plotted.

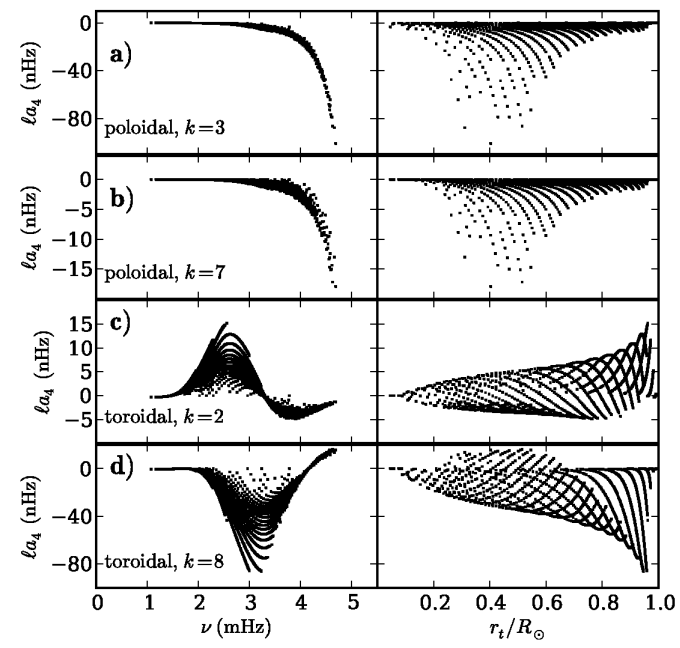

FIG. 6.- Effects of various magnetic fields on the $\ell a_{4}$ splitting coefficients, as a function of both frequency $\nu$ (left hand panels) and lower turning radius $r_{t}$ (right hand panels). The top two panels show the results for poloidal fields with $k=3$ (panel a) and $k=7$ (panel b). The bottom two panels show the results for toroidal fields, both with $\beta_{0}=10^{-4}, r_{0}=0.999 R_{\odot}$ and $d_{0}=0.001 R_{\odot}$. Panel (c) is for a $k=2$ field and panel (d) for a $k=8$ field. Only observed modes have been plotted.

bination of a $k=1$ poloidal field and a near-surface toroidal field $\left(r_{0}=0.999 R_{\odot}, d_{0}=0.001 R_{\odot}\right.$ - the same field from panel (b)). This combination of fields yields a much better fit to this data set, with a reduced $\chi^{2}$ of 2.8. Using a surface toroidal field instead of a poloidal field does not fit the data as well, although it is an improvement over the fit in (b), with a $\chi^{2}$ value of 3.5 . Like the toroidal-only fit, the residuals are more or less without structure in $r_{t}$, but show oscillatory behavior in frequency. The peak field strengths of the two fields are $133 \mathrm{G}$ and $368 \mathrm{G}$ for the poloidal and toroidal fields, respectively. The residuals from this fit can be fit by a toroidal field centered at $r_{0}=0.996 R_{\odot}$, so in panel (d) we show the best fit to the data: a $k=1$ poloidal field with two toroidal fields, one centered at $r_{0}=0.999 R_{\odot}$ and another centered at $r_{0}=0.996 R_{\odot}$. Both toroidal fields are $k=2$ fields and have widths $d_{0}=0.001 R_{\odot}$. The poloidal field has a peak field strength at the surface of $124 \pm 18 \mathrm{G}$, while the toroidal fields have peak field strengths of $380 \pm 30 \mathrm{G}$ and $1.4 \pm 0.2 \mathrm{kG}$, respectively. The reduced $\chi^{2}$ of this fit is 1.7 . Attempts to fit the data with a single toroidal field which occupies the same region as the two field in this fit did not yield a good fit - the data seem to require a double peaked field.

Figure 8 shows the $a_{4}$ splitting coefficients for two different data sets (\#2224 and \#3160), and the same models shown in Fig. 7 panel (d). The errors on the data are large compared to the signal - thus the normalized residuals are comparable to those for the $a_{2}$ coefficients, but some other field configurations also fit the $a_{4}$ coefficients equally well, so we do not use them to constrain the field configurations or determine the field strengths.

Having fit the high activity set, we repeat the fits for all 56 sets in our study. Figure 9 shows fits of the same $k=1$ poloidal plus toroidal field combination to six representative mode sets, covering the rise and fall of solar cycle 23. The first set, \#1216, is the first 72-day mode set from the MDI program, and is a low activity set. Two rising phase sets are shown, \#2224 and \#2728, with $10.7 \mathrm{~cm}$ fluxes of 131.4 SFU and 187.3 SFU, respectively. The high activity set from Fig. 7 is shown, and a declining phase set $\left(\# 3952, F_{10.7}=126.4\right)$ and a set from the current minimum (\#4744). For all sets, the same combination of poloidal and toroidal fields - but with differing field strengths - was found to be the best fit. The residuals show the same structure with frequency.

No mode set in our study is well fit by any magnetic field at the base of the convection zone. For low activity sets (10.7 cm flux of less than $100 \mathrm{SFU})$, we can find an upper bound on the field that could be present in the data. For a field centered at the base of the convection zone with $k=2$ and $d_{0}=0.01 R_{\odot}$, we find that a fields of up to $300 \mathrm{kG}$ can be fit to the data, although this is an upper limit, not a detection, since the models of that field strength or lower give the same $\chi^{2}$ as a zero field strength model. At high activity, the dominant signal is from the surface, which we have attempted to explain with magnetic fields located in those layers.

The field strengths of the poloidal and shallow toroidal field fits to all 56 sets used in this study are shown in Fig. 10. Also shown in this figure is the ratio of the poloidal field strength to the $r_{0}=0.999 R_{\odot}$ toroidal field strength. With the exception of the low activity sets at the beginning and end of the solar cycle, where the uncertainty in the fits is relatively large, the ratio between the poloidal and toroidal field strengths is roughly constant. The field strengths from Fig. 10 are correlated with global activity indices from solar cycle 23 . The correlation coefficients are $0.90,0.93$, and 0.92 for the poloidal and two toroidal field components, respectively. In Fig. 11, we plot the toroidal and poloidal field strengths as a function of one such global index, the $10.7 \mathrm{~cm}$ radio flux. The field strengths prove to be highly correlated with activity, although there is a hysteresislike effect evident in the toroidal field strengths - the rising phase (shown in blue) is weaker than the declining phase fields. The same effect may also be present at low activity in the poloidal field strengths. The poloidal 

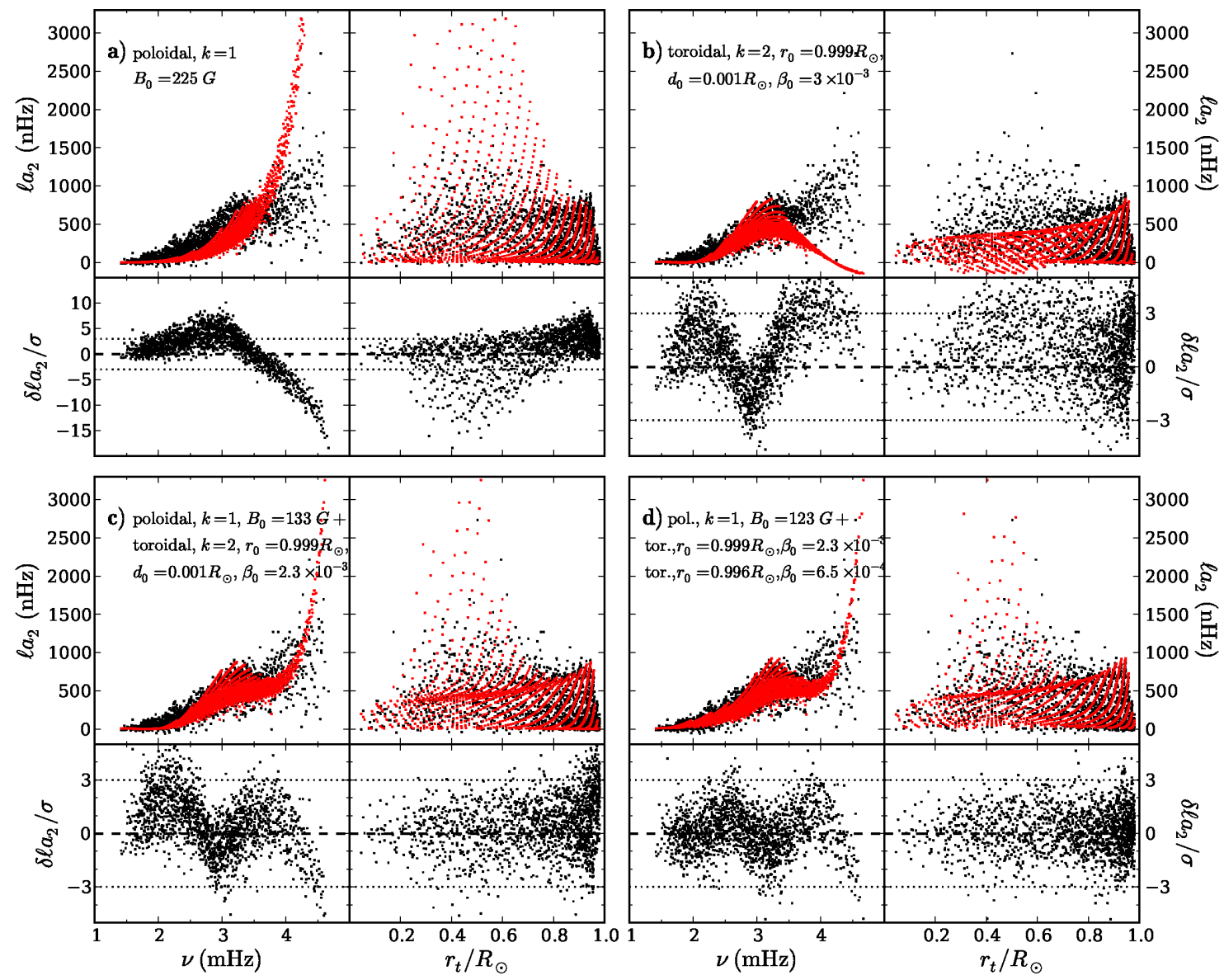

FIG. 7.- Fits to observed splitting coefficients $\ell a_{2}$ for different magnetic field configurations. Four different fits are shown, both as a function of frequency $\nu$ and as a function of lower turning radius $r_{t}$. The data are shown in black, and the modeled points in red. The residuals, scaled by the errors in the data, are also shown below the comparisons. The data are the $\ell a_{2}$ splitting coefficients from an MDI 72 day mode parameter set, taken at the peak of solar cycle 23 (MDI set \#3160, start day 2001 Aug 27). Panel (a) shows a fit from a $k=1$ poloidal field. Panel (b) shows the fit from a near-surface toroidal field. Panel (c) shows the best fit field combination with two fields to this data set - a combination of a dipole poloidal field with a toroidal field located just below the surface $\left(r_{0}=0.999 R_{\odot}, d_{0}=0.001 R_{\odot}\right)$. Panel (d) shows the best fit with three fields - the same poloidal and toroidal field as in panel (c) (though with slightly different field strengths) and another toroidal field at $r_{0}=0.996 R_{\odot}$ and a width of $d_{0}=0.001 R_{\odot}$.

field strengths do seem to saturate at high activity. The strengths of the two toroidal fields are extremely well correlated.

\section{DISCUSSIONS AND CONCLUSIONS}

We have attempted to use the first even order splitting coefficient $\left(a_{2}\right)$ to infer the configuration and strength of the Sun's internal magnetic fields over the course of solar cycle 23, assuming that the entire signature in $a_{2}$ after correction for rotation effects is magnetic and that the fields are axisymmetric. The field that we have found is a combination of poloidal field and a double-peaked nearsurface toroidal field. The strengths of the poloidal and toroidal components, at least for high activity period, are well correlated. The relative strengths of the two toroidal fields are also extremely well correlated.

Although the fits we have shown are the best fit to the data from the grid of models that we have computed, we can say nothing about the uniqueness of these fits over the set of all possible magnetic field configurations in the solar interior. In particular, the choice of radial profile of the toroidal fields is virtually limitless, and by restrict- ing our work to profiles of the form (2), we have limited our search to a restricted class of fields. It is possible that there are fields we did not consider with quite different radial and latitudinal distributions which fit the data as well as the fields we have presented as best fits. In addition, as noted above, it is not strictly correct to add the splitting coefficient perturbations together as we have done without explicitly accounting for the perturbations arising from the cross terms. We do not expect, however, these corrections to be significant, and a full treatment of these corrections would be considered in a future work.

Our inferred magnetic field does not change its latitudinal distribution over the course of the solar cycle. This is in part due to the fact that we are only fitting the $a_{2}$ coefficient (as noted above, the higher order splittings had large errors), so our sampling of the interior is not really latitudinally sensitive. Thus, we do not see a butterfly diagram in our magnetic fields. Ulrich \& Boyden (2005) measured the surface toroidal component of the solar magnetic field over almost an entire 22-year cycle. The field they measure is roughly a tenth of the peak 


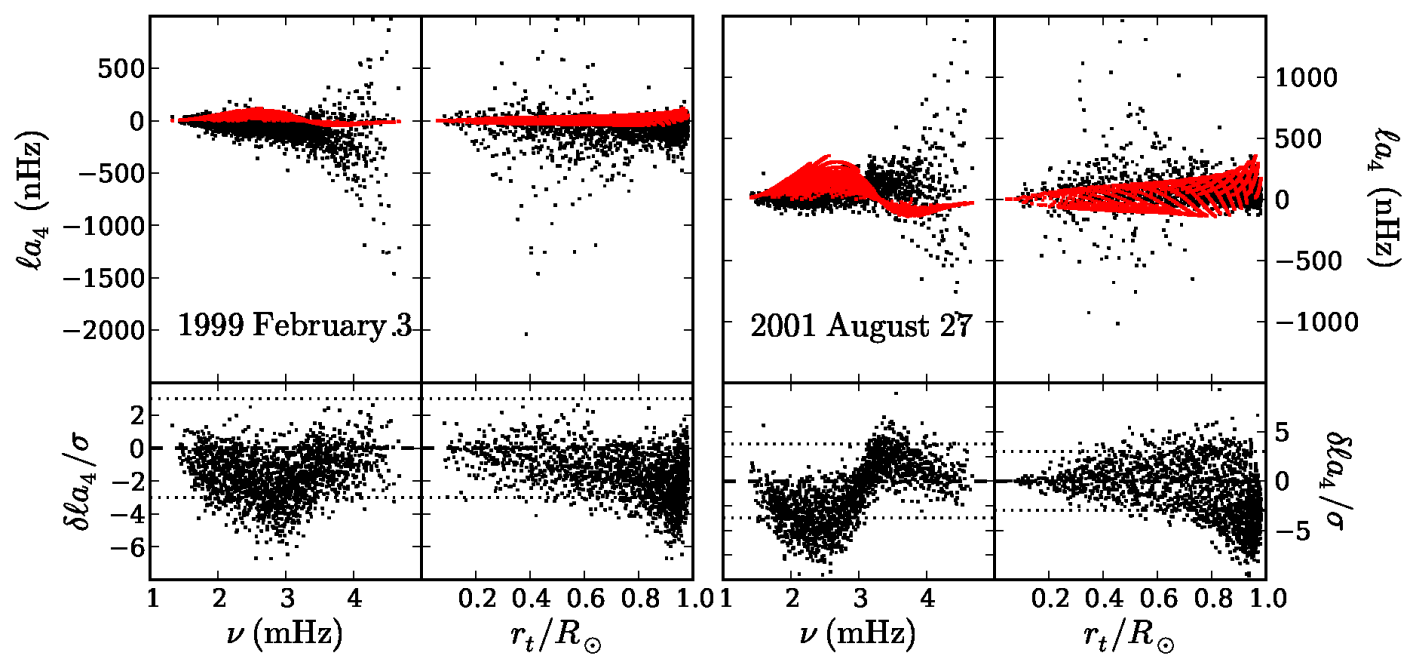

FIG. 8.- Comparisons of data to models for $\ell a_{4}$ splitting coefficients from two different data sets. The field configurations are the same as from Fig. 7 (a). The left hand panels are from set \#2224, the right hand panels are from \#3160. As in the previous figure, data and model are shown both as a function of frequency $\nu$ and as a function of lower turning radius $r_{t}$. The residuals are shown below the data, and are normalized by the errors in the data. The model is obtained by fitting only the $a_{2}$ coefficients.
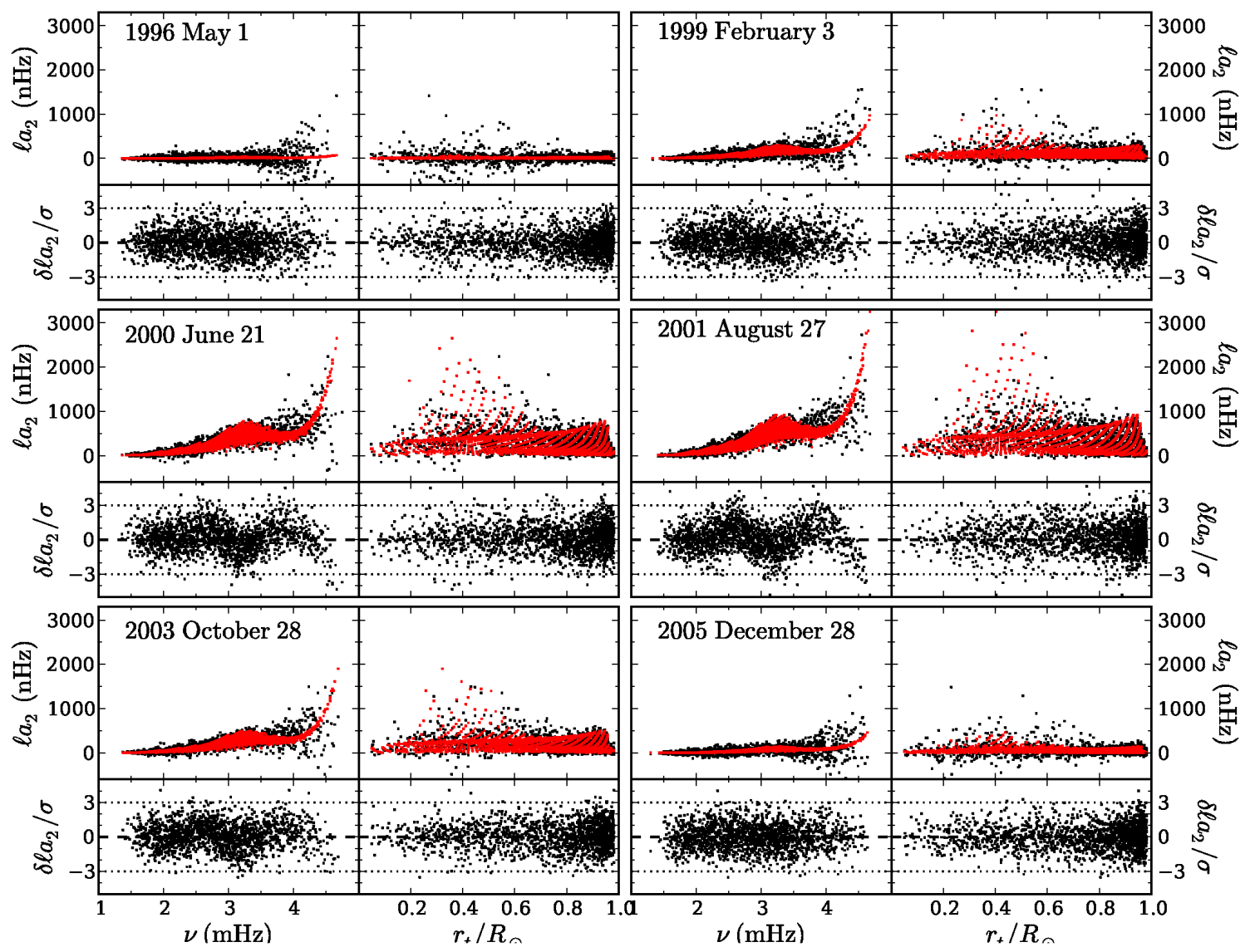

FIG. 9. - Fits to measured splitting coefficients $\ell a_{2}$ for six different sets throughout solar cycle 23. The data (shown in black) are from MDI 72 day mode parameter sets. The magnetic field configuration is the same as panel $\mathbf{d}$ in Figure 7 a dipole poloidal field and two toroidal fields at $r_{0}=0.996 R \odot$ and $r_{0}=0.999 R \odot$, with $d_{0}=0.001 R \odot$ and $k=2$. The fits are shown both as a function of frequency $\nu$ and as a function of lower turning radius $r_{t}$. The residuals scaled by the errors in the data are also shown. The toroidal field strengths at $r=0.999 R_{\odot}$ correspond to $\beta_{0}=10^{-4}, 7.8 \times 10^{-4}, 2 \times 10^{-3}, 2.5 \times 10^{-3}, 1.3 \times 10^{-3}$, and $5 \times 10^{-4}$ for the six sets, respectively. The toroidal field strengths at $r=0.996 R \odot$ correspond to $\beta_{0}=1.2 \times 10^{-5}, 2 \times 10^{-4}, 5.6 \times 10^{-4}, 6.6 \times 10^{-4}, 2.9 \times 10^{-4}$, and $1.4 \times 10^{-4}$. The poloidal field strengths at the surface are $B=0 \mathrm{G}, 68 \mathrm{G}, 115 \mathrm{G}, 125 \mathrm{G}, 94 \mathrm{G}$, and $58 \mathrm{G}$. 


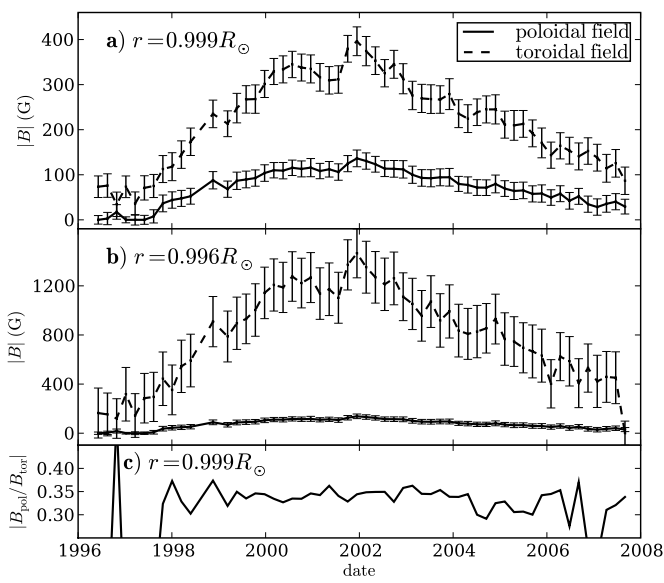

FIG. 10. - The strength of the inferred magnetic fields as a function of time over solar cycle 23. Each MDI 72 day mode parameter set is fitted by with the same magnetic field configuration as Fig. 9 The strengths (in Gauss) of the poloidal field at $r=0.999 R_{\odot}$ (solid black line) and the toroidal field at $r=0.999 R_{\odot}$ (dashed line) are shown in the upper panel (a). The middle panel (b) shows the same quantities as in the upper panel, but this time at a radius of $r=0.996 R_{\odot}$. The lower panel (c) shows the ratio of the poloidal field strength to the toroidal field strength at $r=0.999 R \odot$. The ratio of poloidal to toroidal at $r=0.996 R_{\odot}$ looks very similar.

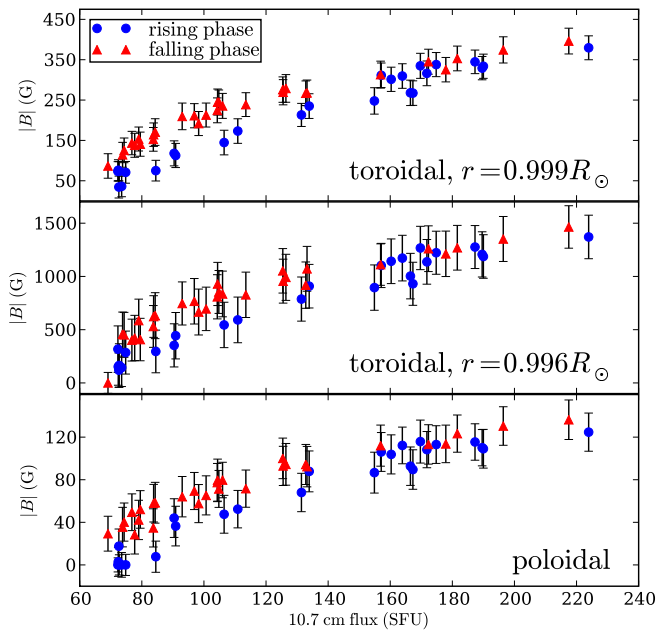

FIG. 11. - The strength of the inferred magnetic fields as a function of $10.7 \mathrm{~cm}$ radio flux. The top panel shows the $r_{0}=0.999 R_{\odot}$ toroidal field strength, the middle panel shows the $r=0.996 R_{\odot}$ toroidal field strength, and the bottom panel shows the poloidal field strength at $r=0.999 R_{\odot}$. Rising and declining phase are distinguished with blue circles for the rising phase and red triangles for the declining phase. The toroidal field shows a hysteresis effect. The poloidal field shows some hysteresis at low activity, as well as a hint of saturation at high activity.

strength of our toroidal field. Strictly speaking, however, we see no toroidal field at all at the surface, since in our inferred field, the field strength becomes zero precisely at $r=1 R_{\odot}$. The peak strength that we measure, however, is only $700 \mathrm{~km}$ below the surface, and the field could penetrate the surface somewhat. Ulrich \& Bovden (2005) find a field which gives a $\beta \sim 6 \times 10^{-5}$ at the surface at high activity, and drops to nothing at low activity, while we find a field that changes from $\beta_{0} \sim 10^{-4}$ at low activity to $\beta_{0}=2 \times 10^{-3}$ at high activity at a radius of $r=0.999 R_{\odot}$ (a depth of approximately $\left.700 \mathrm{~km}\right)$.

Recently, attention has been focused on the strength and configuration of the quiet Sun surface magnetic field. Harvey et al. (2007) reported the presence of a 'seething' horizontal magnetic field with an rms field strength of 1.7 G. With the launch of Hinode (Solar B), the high spatial resolution of the onboard spectropolarimeter has been used to study the horizontal fields of the solar photosphere. Lites et al. (2007, 2008) have measured the horizontal flux, which they find to be $55 \mathrm{G}$, compared to the average vertical flux of $11 \mathrm{G}$. Petrie \& Patrikeeva (2009) found that the zonal component (component in the East-West direction) was much smaller than the radial component, reporting an inclination angle of less than $12^{\circ}$ from vertical in the East-West direction.

The fields being studied in the aforementioned works are generally very tangled fields which thread through the intergranular lanes and so they are not axisymmetric fields. It is worthwhile to compare our results with theirs, since tangled fields on local scales can organize into roughly axisymmetric fields on global scales. However, the contribution to splittings are more sensitive to $\left\langle B^{2}\right\rangle$ rather than $\langle B\rangle^{2}$ and hence tangled field may also contribute to it, even when the average $\langle B\rangle$ is very small. Further, considering the general behavior of the perturbation to the mode frequencies, our inference about the location of required magnetic field is more robust as a different location will yield a very different behavior of splitting coefficients. The exact magnitude of the field may depend on the assumption of geometry and on it being tangled or large scale. Nevertheless, we believe that our estimate is of the right order, though the statistical errorbars obtained by us may not be realistic. The systematic errors in these estimates would be certainly larger. The dominance of poloidal field orientation at the surface found by Petrie \& Patrikeeva (2009) is found in our own results - at the surface, the toroidal field is weak or vanishing, but the poloidal field remains. In the period analyzed by Lites et al. (2007, 2008), we find a poloidal field strength of $40 \mathrm{G}$, and a toroidal field of $90 \mathrm{G}$ at a depth of $700 \mathrm{~km}$. The vertical flux they find $(11 \mathrm{G})$ is weaker than what we detect, but their $55 \mathrm{G}$ horizontal flux may be roughly consistent with our toroidal field.

Schrijver \& Liu (2008) found that the dipole moment of the surface magnetic field, measured from MDI magnetograms, was half the strength in 2008 that it was in 1997, during the last solar minimum. We do not see such a difference from the beginning of our period to the end - in fact, we find the poloidal field strength is slightly higher during the current minimum, although the level of the difference is within the errors, and our data sets end in 2007, so the comparison is not contemporaneous.

Hysteresis in the relations between activity indices has been observed before, for example in the relation between low degree (Anguera Gubau et al. 1992; Jimenez-Reves et al. 1998) and intermediate degree (Tripathy et al. 2000, 2001) acoustic modes and global magnetic indices. It should be noted that an analysis of a full solar cycle's worth of intermediate degree $p$-modes data does not show any hysteresis in mean frequencies as a function of $10.7 \mathrm{~cm}$ flux (Baldner \& Basu 2008). Tripathy et al. (2001) noted that, among the global mode 
indices, the relation between global line-of-sight magnetic flux and $10.7 \mathrm{~cm}$ radio flux showed a hysteresis effect, but the relation between the radiative indices and $10.7 \mathrm{~cm}$ flux did not. Moreno-Insertis \& Solanki (2000) argued that the observed hysteresis could be almost entirely due to the latitudinal distribution of magnetic flux on the surface of the Sun. We believe that this is a compelling explanation for the hysteresis that we find. The $10.7 \mathrm{~cm}$ flux is the integrated flux received at the Earth and does not contain any information about the latitudinal variation, while the $a_{2}$ splitting coefficient is associated with definite latitudinal variation, given by $P_{2}(\cos \theta)$ (Antia et al. 2001), and hence the two would not be the same. More importantly, we expect the actual magnetic fields in the near surface layers to drift equatorward - as the surface fields do.

Few conclusions can really be drawn from this work with respect to dynamo theory since the fields we have inferred are predominantly shallow fields, whereas most dynamo models operate much deeper down, in the shear layer at and below the base of the convection zone. (some useful recent reviews include Ossendrijver 2003; Charbonneau 2005; Miesch \& Toomre 2009). The upper limits that we place on fields at that depth are consistent with earlier helioseismic results (e.g., Basu 1997; Antia et al. 2000; Chou \& Serebryanskiv 2002,
2005; Baldner \& Basu 2008). Many deep-seated dynamo mechanisms predict an anticorrelation between the poloidal and toroidal field components, as the dynamo converts poloidal to toroidal field and toroidal field back to poloidal. We do not see any evidence of such conversion. Some dynamo mechanisms, however, operate in the near-surface shear layer (e.g. Brandenburg 2005). Although the fields generated in these models are generally extremely tangled, on global scales these fields can have toroidal and poloidal components (e.g. Brown et al. 2007, 2009). In particular, although they were considering a more rapidly rotating star than the Sun, Brown et al. (2007) noted that their field contained both a poloidal and a toroidal component, and that the toroidal component was much the stronger of the two.

This work utilizes data from the Solar Oscillations Investigation/ Michelson Doppler Imager (SOI/MDI) on the Solar and Heliospheric Observatory (SOHO). SOHO is a project of international cooperation between ESA and NASA. MDI is supported by NASA grants NAG58878 and NAG5-10483 to Stanford University. This work was partially supported by NSF grants ATM 0348837 and ATM 0737770 to SB. CB is supported by a NASA Earth and Space Sciences Fellowship NNX08AY41H.

\section{REFERENCES}

Antia, H. M., \& Basu, S. 1994, A\&AS, 107, 421

Antia, H. M., Basu, S., Hill, F., Howe, R., Komm, R. W., \& Schou, J. 2001, MNRAS, 327, 1029

Antia, H. M., Basu, S., \& Chitre, S. M. 2008, ApJ, 681, 680

Antia, H. M., Chitre, S. M., \& Thompson, M. J. 2000, A\&A, 360, 335

Baldner, C. S., \& Basu, S. 2008, ApJ, 686, 1349

Baldner, C. S., Basu, S., \& Larson, T. P. 2008, in ASP

Conf. Ser. GONG 2008/SOHO XXI Meeting on Solar-Stellar

Dynamos as Revealed by Helio-and Asteroseismology, ed.

Dikpati, M., Gonzalez-Hernandez, I., Arentoft, T., \& Hill, F., in press

Basu, S., Antia, H. M., \& Narasimha, D. 1994, MNRAS, 267, 209

Basu, S. 1997, MNRAS, 288, 572

Brandenburg, A. 2005, ApJ, 625, 539

Brown, B. P., Browning, M. K., Brun, A. S., Miesch, M. S., Nelson, N. J., \& Toomre, J. 2007, in AIP Conf. Ser. 948, Unsolved Problems in Stellar Physics: A Conference in Honor of Douglas Gough, ed. Stancliffe, R. J., Houdek, G., Martin, R. G., \& Tout, C. A., 271

Brown, B. P., Browning, M. K., Miesch, M. S., Brun, A. S., \& Toomre, J. 2009, arXiv:0906.2407

Charbonneau, P. 2005, Living Reviews in Solar Physics, 2, 2

Chou, D.-Y., \& Serebryanskiy, A. 2002, ApJ, 578, L157

Chou, D.-Y., \& Serebryanskiy, A. 2005, ApJ, 624, 420

D'Silva, S. \& Choudhuri, A. R. 1993, A\&A, 272, 621

Dziembowski, W., \& Goode, P. R. 1984, Memorie della Societa Astronomica Italiana, 55, 185

Dziembowski, W., \& Goode, P. R. 1988, in IAU Symp. 123, Advances in Helio- and Asteroseismology, ed.

Christensen-Dalsgaard, J. C. D. \& Frandsen, S., 171

Dziembowski, W. A., Goode, P. R., Kosovichev, A. G., \& Schou, J. 2000, ApJ, 537, 1026

Dziembowski, W. A., Goode, P. R., \& Schou, J. 2001, ApJ, 553, 897

Dziembowski, W. A., Pamyatnykh, A. A., \& Sienkiewicz, R. 1990, MNRAS, 244, 542

Gough, D. O. 1990, Lecture Notes in Physics, 367, Progress of Seismology of the Sun and Stars, ed. Osaki, Y. \& Shibahashi, H., 283
Gough, D. O., \& Thompson, M. J. 1990, MNRAS, 242, 25

Anguera Gubau, M., Palle, P. L., Perez Hernandez, F., Regulo, C., \& Roca Cortes, T. 1992, A\&A, 255, 363

Harvey, J. W., Branston, D., Henney, C. J., \& Keller, C. U. 2007, ApJ, 659, L177

Isaak, G. R. 1982, Nature, 296, 130

Jimenez-Reyes, S. J., Regulo, C., Palle, P. L., \& Roca Cortes, T. 1998, A\&A, 329, 1119

Larson, T. P., \& Schou, J. 2008, Journal of Physics Conference Series, 118, 012083

Lites, B. W., et al. 2008, ApJ, 672, 1237

Lites, B., et al. 2007, PASJ, 59, 571

Miesch, M. S., \& Toomre, J. 2009, Annual Review of Fluid Mechanics, 41, 317

Moreno-Insertis, F., \& Solanki, S. K. 2000, MNRAS, 313, 411

Ossendrijver, M. 2003, in ASP Conf. Ser. 286, Current Theoretical Models and Future High Resolution Solar Observations: Preparing for ATST, ed. Pevtsov, A. A. \& Uitenbroek, H., 97

Petrie, G. J. D., \& Patrikeeva, I. 2009, ApJ, 699, 871

Ritzwoller, M. H., \& Lavely, E. M. 1991, ApJ, 369, 557

Roxburgh, I. W., \& Vorontsov, S. V. 1994, MNRAS, 268, 880

Schou, J., et al. 1998, ApJ, 505, 390

Schou, J. 1999, ApJ, 523, L181

Schrijver, C. J., \& Liu, Y. 2008, Sol. Phys., 252, 19

Thompson, M. J., et al. 1996, Science, 272, 1300

Tripathy, S. C., Kumar, B., Jain, K., \& Bhatnagar, A. 2000, Journal of Astrophysics and Astronomy, 21, 357

Tripathy, S. C., Kumar, B., Jain, K., \& Bhatnagar, A. 2001, Sol. Phys., 200, 3

Ulrich, R. K., \& Boyden, J. E. 2005, ApJ, 620, L123

Zweibel, E. G., \& Gough, D. 1995, in ESA SP 376, Helioseismology, ed. Hoeksema, J. T., Domingo, V., Fleck, B., \& Battrick, B., 73 